\title{
Broad Humoral and Cellular Immunity Elicited by a Bivalent DNA Vaccine Encoding HA and NP Genes from an $\mathrm{H} 5 \mathrm{~N} 1$ Virus
}

\author{
Ke Xu, ${ }^{1, \star}$ Zhi-Yang Ling, ${ }^{2, *}$ Liang Sun, ${ }^{2}$ Ying Xu, ${ }^{2}$ Chao Bian,, Yuan He, Wei Lu, Ze Chen, ${ }^{1}$ and Bing Sun ${ }^{1,2}$
}

\begin{abstract}
Influenza A virus is highly variable and a major viral respiratory pathogen that can cause severe illness in humans. Therefore it is important to induce a sufficient immune response specific to current strains and to heterosubtypic viruses with vaccines. In this study, we developed a dual-promoter-based bivalent DNA vaccine that encodes both hemagglutinin (HA) and nucleoprotein (NP) proteins from a highly pathogenic A/Chicken/ Henan/12/2004 (H5N1) virus. Our results show that the expression levels of HA and NP genes from the dualpromoter plasmid are similar to those seen when they are expressed individually in independent plasmids. When the bivalent DNA vaccine was inoculated via intramuscular injection and in vivo electroporation, high levels of both humoral and cellular immune responses were elicited against homologous H5N1 virus and heterosubtypic H9N2 virus. Furthermore, no obvious antigenic competition was observed between HA and NP proteins in the dual-promoter-based bivalent vaccine compared to monovalent vaccines. Our data suggest that a combination of influenza surface and internal viral genes in a dual-promoter-expressing plasmid may provide a new approach for developing a DNA vaccine that may protect not only specifically against a currently circulating strain, but also may cross-protect broadly against new heterosubtypic viruses.
\end{abstract}

\section{Introduction}

$\mathbf{T}$ He AVIAN INFLUENZA ViRUSES WERE THOUght UNABLE to be transmitted directly from birds to humans until 1997, when a highly pathogenic avian influenza (HPAI) A virus H5N1 broke out in Hong Kong $(12,16,40)$. Data from the World Health Organization (WHO) indicated 500 confirmed human cases of H5N1 virus infection, and the fatality rate for the virus is approximately $60 \%$ (50). In addition to the H5N1 virus, other avian influenza virus subtypes have also been reported to cause human infections $(4,31,54)$. Although the slaughter of poultry efficiently eliminated the source of the infection, a human pandemic caused by an avian virus is still possible (6). Furthermore, potential reassortment between human flu viruses and avian flu viruses has caused global concern about the possibility of creating a new virulent strain that is more easily transmissible and lethal to humans (36). Therefore, vaccines that protect against infection or limit the spread of avian influenza viruses, particularly HPAI viruses, are urgently needed.

Influenza A virus has been successful in its evolution due to its antigenic variation, which appears in two forms: antigenic shift and antigenic drift. This epidemiological property may cause the emergence of new epidemic and pandemic viruses annually. As a result, every year before the ensuing influenza season, the WHO recommends the composition of an influenza vaccine suitable for the upcoming year based on global surveillance. However, the seasonal vaccine reconstituted with the WHO-recommended strains may not always be capable of providing protection against a new emerging epidemic strain. The 2009 swine-origin influenza virus (S-OIV) H1N1 is such a case, and it broke out in April 2009 in Mexico and the U.S. $(7,23,51)$. Analysis of its antigenic and genetic characteristics showed that this virus was a new reassortant virus introduced in humans, against which the population had little immunity $(25,52)$. Vaccines against

\footnotetext{
${ }^{1}$ Molecular Virus Unit, Key Laboratory of Molecular Virology and Immunology, Institut Pasteur of Shanghai, Chinese Academy of Sciences, Shanghai, P.R. China.

${ }^{2}$ Laboratory of Molecular Cell Biology, Institute of Biochemistry and Cell Biology, Shanghai Institutes for Biological Sciences, Chinese Academy of Sciences, Shanghai, P.R. China.

${ }^{3}$ Shanghai Institute of Biological Products, Shanghai, P.R. China.

*Both of these authors contributed equally to this work.
} 
this novel virus were urgently produced worldwide to prevent a potential epidemic in the winter season. It is consequently believed that an influenza vaccine candidate that provides broad cross-strain protection is a promising way to deal with such situations in the future, and may provide protection against this ever-changing virus (18).

Thus an ideal vaccine against influenza virus must be not only specific to the currently circulating strain, but must also cover different subtypes. The most widely used influenza vaccines, the inactivated vaccines, fail to fulfill this criterion, as they are only able to elicit antibody responses against viral proteins, and thus are only effective for circulating strains (34). The live attenuated vaccines, including cold adapted vaccines and genetically engineered vaccines, appear to be ideal, as they can induce both antibody responses against circulating strains, and a cell-mediated immune response against internal viral proteins conserved among subtypes. However, a potential reassortment with natural virus increases the risk of this approach (34). In addition, the handling of live viruses needed to make these vaccines restrict its large-scale production. Thus the developing DNA vaccine approach is promising due to induction of both humoral and cellular immune responses, and its safety and low cost of production $(19,21)$.

As a model system for DNA vaccines, influenza DNA vaccines have been proven in different animal models to provide protective immunity against viral infection (44). DNA constructs expressing the viral surface glycoproteins hemagglutinin (HA) and neuraminidase (NA) were demonstrated to provide efficient protection against homologous or drift viral infection, but little cross-subtype protection was elicited $(10,29,30,37,45)$. Plasmids encoding internal conserved viral proteins, for example nucleoprotein (NP) and matrix protein 1 (M1), are able to elicit protection against heterosubtypic virus challenge via a cell-mediated immune response $(19,20,46,47)$. It is therefore reasonable to propose that a combination of the surface and internal antigens of influenza virus may provide effective protection, not only against the circulating strain, but also broadly against heterosubtypic viruses.

In this study, we generated an effective bivalent DNA vaccine by encoding both HA and NP proteins from the HPAI virus A/Chicken/Henan/12/2004 (H5N1). The results show that this bivalent vaccine can elicit humoral and cell-mediated immune responses against both homologous and heterosubtypic viruses when administered via intramuscular (IM) injection and in vivo electroporation. Our work provides a new DNA vaccine candidate that is not only specific enough to neutralize HPAI H5N1 virus, but is also broadly responsive to heterosubtypic viruses.

\section{Materials and Methods}

\section{Mice and viruses}

Female BALB/c mice 4-6 weeks old were purchased from Shanghai SLAC Laboratory Animal Co., Ltd., and randomly divided into groups of 10 mice per group. All mice were maintained with free access to sterile food and water.

The A/Chicken/Henan/12/2004 (H5N1) virus inactivated with formaldehyde was kindly provided by Prof. Ze Chen (Shanghai Institute of Biological Products). A/ Chicken/Jiangsu/7/2002 (H9N2) virus growing in Madin-
Darby canine kidney cells was collected and inactivated with formaldehyde.

\section{Plasmid constructs for immunization}

Viral cDNA from A/Chicken/Henan/12/2004 (H5N1) was kindly provided by Prof. Ze Chen. The full-length HA gene was cloned into the pBudCE4.1 vector (Invitrogen, Carlsbad, CA), under the control of the human elongation factor $1 \alpha$-subunit $(\mathrm{EF}-1 \alpha)$ promoter, using the restriction enzyme NotI/XholI (Takara Bio Inc., Shiga, Japan), and was designated pHA. The full-length NP gene was cloned into the pBudCE4.1 vector under the control of the human cytomegalovirus (CMV) immediate-early promoter using restriction enzyme BamHI/HindIII (Takara Bio Inc.), and was designated pNP. The full-length NP gene was further cloned into $\mathrm{pHA}$ under the CMV promoter to generate $\mathrm{pHA} / \mathrm{NP}$.

To produce $\mathrm{H} 5 \mathrm{~N} 1$ pseudotyped virus, the NA gene from A/Chicken/Henan/12/2004 (H5N1) was cloned into pHA under the CMV promoter, and was designated pHA/NA. Plasmids were grown in DH- $5 \alpha$ E. coli strain, and purified using commercial purification kits. All plasmids were confirmed by sequence analysis.

\section{Cells and transfection}

Human embryonic kidney 293T cells (HEK 293T; ATCC), and Madin-Darby canine kidney cells (MDCK; ATCC) were cultured in Dulbecco's modified Eagle's medium (DMEM) (Gibco, Carlsbad, CA) containing 10\% fetal bovine serum (Gibco) at $37^{\circ} \mathrm{C}$ in a $\mathrm{CO}_{2}$ incubator.

Lipofectamine (Invitrogen) was applied for transient transfection following the manufacturer's protocol.

\section{Western blot analysis}

Cells were lysed directly in sodium dodecyl sulfate (SDS) sample buffer $(60 \mathrm{mM}$ Tris-HCl [pH 6.8], 2\% SDS, $10 \%$ glycerol, 5\% 2-mercaptoethanol, and $0.01 \%$ bromophenol blue), followed by boiling for $10 \mathrm{~min}$. Whole-cell lysates were further subjected to SDS-PAGE. The proteins were transferred to nitrocellulose membranes (BioRad Laboratories, Inc., Hercules, CA). The HA protein was detected with antiHA polyclonal antibody (Antibody Research Centre, Shanghai Institute for Biological Sciences). NP protein was detected with anti-NP polyclonal antibody (Antibody Research Centre, Shanghai Institute for Biological Sciences), and actin was detected with rabbit anti-actin polyclonal antibody (Sigma-Aldrich, St. Louis, MO). Goat anti-rabbit IgG HRP-conjugated antibody (Southern Biotechnology Associates, Birmingham, AL) was applied as the secondary antibody. The exposure was developed with an enhanced chemiluminescence kit (Pierce Protein Research Products, Rockford, IL).

\section{Immunization by in vivo electroporation}

Plasmid DNA encoding viral proteins was inoculated by in vivo electroporation, which was carried out according to the method previously described (1). Mice (aged 4-6 wk) were immunized thrice (at 3-wk intervals) by injection into the quadriceps muscles of two legs with the same molar amount of the vector (pBudCE4.1), $\mathrm{pHA}, \mathrm{pNP}$, or $\mathrm{pHA} / \mathrm{NP}$, at doses of 50,65, 63, and $83 \mu \mathrm{g}$ per mouse, respectively. A 
pair of 5-mm-separated electrode needles was then inserted into the muscle to cover the DNA injection sites, and electric pulses were delivered using an electric pulse generator (Electro Square Porator T830 M; BTX, San Diego, CA) $(8,15)$. In vivo electroporation parameters were: $100 \mathrm{~V}, 5$ pulses, $20 \mathrm{msec}$ pulse duration, and $200 \mathrm{msec}$ between pulses.

\section{Detection of anti-influenza-virus antibody}

Antibody levels against H5N1 (A/Chicken/Henan/12/ 2004) and H9N2 (A/Chicken/Jiangsu/7/2002) were assayed by enzyme-linked immunosorbent assay (ELISA). In brief, 96-well microtiter plates were coated with $5 \mu \mathrm{g} / \mathrm{mL}$ of chemically-killed corresponding virus at $4^{\circ} \mathrm{C}$ overnight. Following the blocking of each well with $10 \%$ bovine serumPBST at $37^{\circ} \mathrm{C}$ for $2 \mathrm{~h}$, the mouse sera collected on the indicated days were prepared in serial dilutions, and were added to each well and incubated at $37^{\circ} \mathrm{C}$ for $2 \mathrm{~h}$. A secondary goat anti-mouse IgG HRP-conjugated antibody (Sigma-Aldrich) at 1:1000 dilution was added to each well and incubated at $37^{\circ} \mathrm{C}$ for $1 \mathrm{~h}$. To develop the ELISA, a 10-mg TMB tablet (SigmaAldrich) was dissolved in $1 \mathrm{~mL}$ of $0.025 \mathrm{M}$ phosphate-citrate buffer, and $50 \mu \mathrm{L}$ of the resulting solution was added to each well. The reaction was stopped by the addition of $0.2 \mathrm{M}$ $\mathrm{H}_{2} \mathrm{SO}_{4}$, and the resulting optical density (OD) at $450 \mathrm{~nm}$ was analyzed with a plate reader.

\section{Production of H5N1 pseudotyped virus and neutralization assay}

Pseudotyped virus was produced to avoid the manipulation of live HPAI virus in the neutralization assay as previously described $(3,11)$. Briefly, HEK293T cells were plated at approximately $50 \%$ confluence in six-well plates. Twenty-four hours later, the plasmid pHA/NA encoding the glycoproteins HA and NA from A/Chicken/Henan/12/2004 (H5N1) virus was co-transfected with packaging plasmid pNL4$3^{\text {Luc+Env-Vpr- }}$ to generate a pseudotyped HIV-backbone luciferase-reporter virus with influenza glycoproteins embedded on the surface. Supernatants from the HEK293T cells were collected at $48 \mathrm{~h}$ post-transfection and aliquotted after clarification by low-speed centrifugation ( $3000 \mathrm{rpm}$ for $5 \mathrm{~min}$ ), and kept frozen.

For the neutralization assay, MDCK cells were plated as target cells in 24-well plates. During infection, $100 \mu \mathrm{L} \mathrm{H5N1}$ pseudotyped virus was incubated with twofold serial dilutions of antisera (starting dilution 1:500) in $900 \mu \mathrm{L}$ DMEM at $37^{\circ} \mathrm{C}$ for $1 \mathrm{~h}$. Subsequently, the medium was aspirated and $1 \mathrm{~mL}$ virus-antiserum mixture was transferred onto the cells. Twenty-four hours later, $1 \mathrm{~mL}$ fresh DMEM without FBS was added, and the cells were lysed with passive lysis buffer (Promega Corp., Madison, WI) 48h later. Because the pseudotyped virus is env ${ }^{-}$, it is competent for only a single cycle of virus replication. Thus the intracellular amount of luciferase activity represents a direct reflection of virus entry. Luciferase assay was performed with Steady-Glow Luciferase Substrate (Promega Corp.).

\section{Splenocyte proliferation activity assay}

On the seventh day after the third injection, the mice were sacrificed and a single-cell suspension was prepared from the spleens of each group. The splenocytes were cultured in triplicate in 96-well plates at $4 \times 10^{5}$ cells per well in RDF medium (Gibco) with 10\% fetal bovine serum (Gibco). The cultures were then stimulated for $36 \mathrm{~h}$ with each of the following: $10 \mu \mathrm{g} / \mathrm{mL}$ BSA (irrelevant antigen), $2 \mu \mathrm{g} / \mathrm{mL}$ Con A (positive control), and $10 \mu \mathrm{g} / \mathrm{mL}$ of the chemicallykilled $\mathrm{H} 5 \mathrm{~N} 1$ virus, or $10 \mu \mathrm{g} / \mathrm{mL}$ of the chemically-killed $\mathrm{H} 9 \mathrm{~N} 2$ virus. The cells were then incubated at $37^{\circ} \mathrm{C}$ and $5 \%$ $\mathrm{CO}_{2}$ for $36 \mathrm{~h}$, then $1 \mu \mathrm{Ci}{ }^{3}[\mathrm{H}]$ thymidine (Amersham Pharmacia Biotech, Pistcataway, NJ) was added to each well for $8-12 \mathrm{~h}$. The cells were harvested and ${ }^{3}[\mathrm{H}]$ thymidine uptake (cpm) was counted on a Beckman LS 6500 scintillation counter (Beckman Coulter, Fullerton, CA). The stimulation indexes (SI) were determined by cpm reading of antigencontaining wells divided by cpm reading of wells without antigen.

\section{In vivo $C T L$ assay}

The in vivo cytotoxicity assay was performed as previously documented $(13,33)$. To prepare target cells, splenocytes were obtained from naïve BALB/c spleen cell suspensions after erythrocytes were removed. The cells were then washed and split into two populations. One population was pulsed with $10^{-6} \mathrm{M}$ of a $1: 1$ peptide mixture (HA:IYSTVASSL or NP:TYQRTRALV) (17), incubated at $37^{\circ} \mathrm{C}$ in $5 \% \mathrm{CO}_{2}$ for $1 \mathrm{~h}$, washed, labeled with a high concentration of CFSE (carboxyfluorescein diacetate $N$-succinimidyl ester; SigmaAldrich) $(5 \mu \mathrm{M})$, and designated CFSE ${ }^{\text {high }}$ cells. The other population was left without peptide, labeled with a low concentration of CFSE $(0.5 \mu \mathrm{M})$, and designated CFSE ${ }^{\text {low }}$ cells. For in vivo injection, a mixture of equal numbers of cells from the CFSE ${ }^{\text {high }}$ and CFSE $^{\text {low }}$ populations $\left(2 \times 10^{7}\right.$ cells in $400 \mu \mathrm{L}$ of PBS in total) was injected intravenously into each immunized mouse on the seventh day after the third immunization. Four hours after injection, the mice were sacrificed for their splenocytes. Cell suspensions were analyzed on a FACSCalibur analyzer (BD Biosciences Pharmingen, Franklin Lakes, NJ), and each population was detected by its differential CFSE fluorescence intensity. Up to $1 \times 10^{4}$ CFSEpositive cells were collected for analysis. To calculate specific lysis, the following formulas were used: ratio = (percentage $\mathrm{CFSE}^{\text {low }} /$ percentage $\left.\mathrm{CFSE}^{\text {high }}\right)$; percentage specific lysis= $[1 \times($ ratio unprimed/ratio primed $) \times 100](27)$.

\section{Cytokine assay}

On the seventh day after the third immunization, splenocytes were harvested for antigen restimulation. First, $4 \times 10^{5}$ cells in 96-well plates were stimulated in culture with $10 \mu \mathrm{g} / \mathrm{mL}$ BSA (irrelevant antigen), $2 \mu \mathrm{g} / \mathrm{mL}$ ConA (positive control), $10 \mu \mathrm{g} / \mathrm{mL}$ chemically-killed $\mathrm{H} 5 \mathrm{~N} 1$ virus, or $10 \mu \mathrm{g} /$ $\mathrm{mL}$ chemically-killed $\mathrm{H} 9 \mathrm{~N} 2$ virus, at $37^{\circ} \mathrm{C}$ in $5 \% \mathrm{CO}_{2}$ for $72 \mathrm{~h}$. The levels of secreted IFN- $\gamma$ and IL- 4 in the cultured supernatant were determined using ELISA kits from R\&D Systems (Minneapolis, MN).

\section{Data analysis}

All values were expressed as mean \pm standard deviation (SD). Statistical analysis of the experimental data was performed using the two-tailed Student's t-test or one-way analysis of variance (ANOVA). For all tests, $p<0.05$ was considered statistically significant. 


\section{Results}

\section{Expression of DNA constructs in eukaryotic cells}

To test whether a bivalent DNA vaccine containing HA and NP from an HPAI H5N1 virus could induce both strong cellular and humoral immunity, the entire length of the HA and NP coding regions from A/Chicken/Henan/12/2004 (H5N1) virus were cloned into a dual-promoter eukaryotic-expressing vector $\mathrm{pBudCE} 4.1$ under their respective promoters as shown in Fig. 1A. The pHA/NP plasmid expressing two viral proteins at the same time was used as the bivalent DNA vaccine, while the monovalent plasmids $\mathrm{pHA}$ and $\mathrm{pNP}$, each encoding only one viral protein, and vector plasmid pBudCE4.1, were included as controls. To confirm the expression of all the DNA constructs, human 293T cells were transfected with same molar amount of the corresponding plasmid, and protein expression was analyzed by Western blot assay with anti-HA or anti-NP polyclonal antibody. As shown in Fig. 1B, the coexpression of HA and NP in pHA/NP was similar to the levels of pHA or pNP expressed singly. These data suggest that the bivalent DNA vaccine was able to express successfully and efficiently in eukaryotic cells.

\section{HA expression triggers a predominantly humoral} immune response with neutralizing activity, and NP elicits a cross-response to heterosubtypic virus

To examine the humoral response induced by the bivalent DNA vaccine, we first examined the level of anti-H5N1- specific antibodies in mice immunized with the respective DNA constructs. Sera of vaccinated mice from days 21, 35, and 49 post-initial immunization were assayed by ELISA, and the titers of anti-H5N1-specific IgG were analyzed. The data in Fig. 2A show that mice immunized with pHA, pNP, and $\mathrm{pHA} / \mathrm{NP}$ induced significantly high levels of specific antibodies against chemically-killed H5N1 virus, compared to the vector-immunized group at day 49. Moreover, the highest antibody titer was observed in the group immunized with the bivalent DNA plasmid pHA/NP, which was proposed to elicit both anti-HA and anti-NP antibodies.

As neutralizing antibody is responsible for efficiently preventing virus infection, we further examined the neutralizing activity of the antisera. To this end, H5N1pseudotyped virus was obtained by co-expression of the viral HA and NA genes on the surface of a HIV-based packaging virion. The virus-neutralizing abilities of the sera from all groups were assessed using reductions in luciferase expression of the H5N1-pseudotype virus (3). The results clearly showed that of all immunized groups, only the $\mathrm{pHA}$ and pHA/NP groups were able to induce a strong neutralizing-antibody response (Fig. 2B). In contrast, little neutralizing antibody was elicited in the group immunized with $\mathrm{pNP}$ or vector, indicating that the HA protein of influenza virus is the predominant inducer of neutralizing antibody.

To test whether our bivalent DNA vaccine had broad response against heterosubtypic virus, we then examined the heterosubtypic IgG against avian influenza subtype H9N2 virus, which is also able to cause cross-species infection in hu-

A
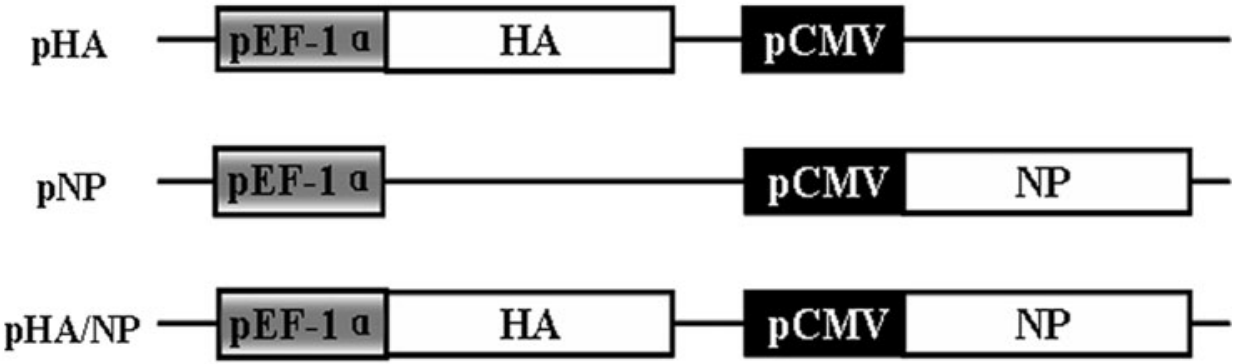

B

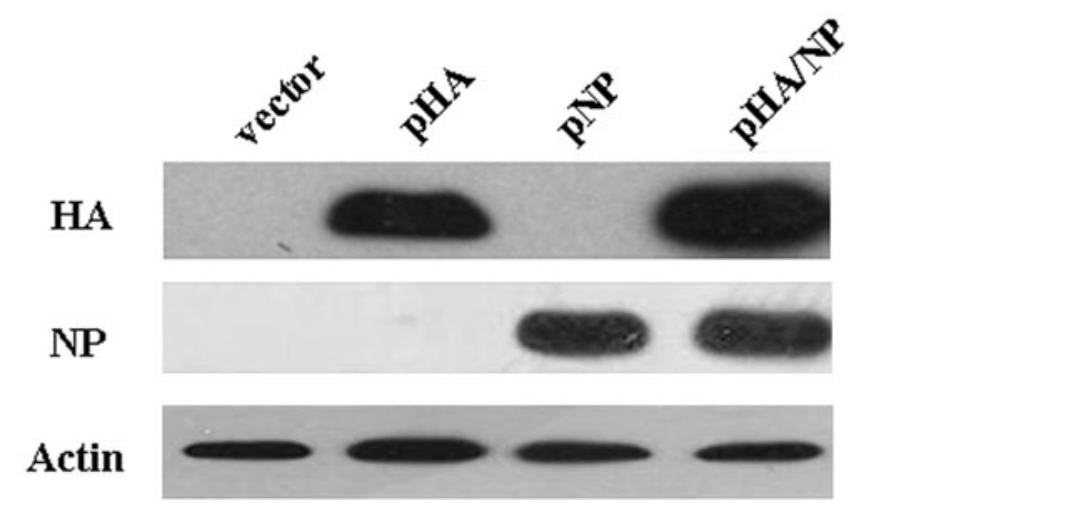

FIG. 1. Construction and eukaryotic expression of DNA vaccines. (A) Schematic diagram of DNA vaccine constructs. The full-length HA and NP genes of H5N1-HPAIV strain A/Chicken/Henan/12/2004 was cloned into the dual-promoter plasmid pBudCE4.1 for eukaryotic expression. (B) Expression of DNA constructs in HEK293T cells. The HEK293T cells were transfected with equal molar amounts of the indicated plasmids, and cell lysates were collected $24 \mathrm{~h}$ post-transfection and subjected to Western blot analysis with anti-HA and anti-NP polyclonal antibodies. The $\beta$-actin was detected with anti-actinspecific polyclonal antibody as a loading control. 

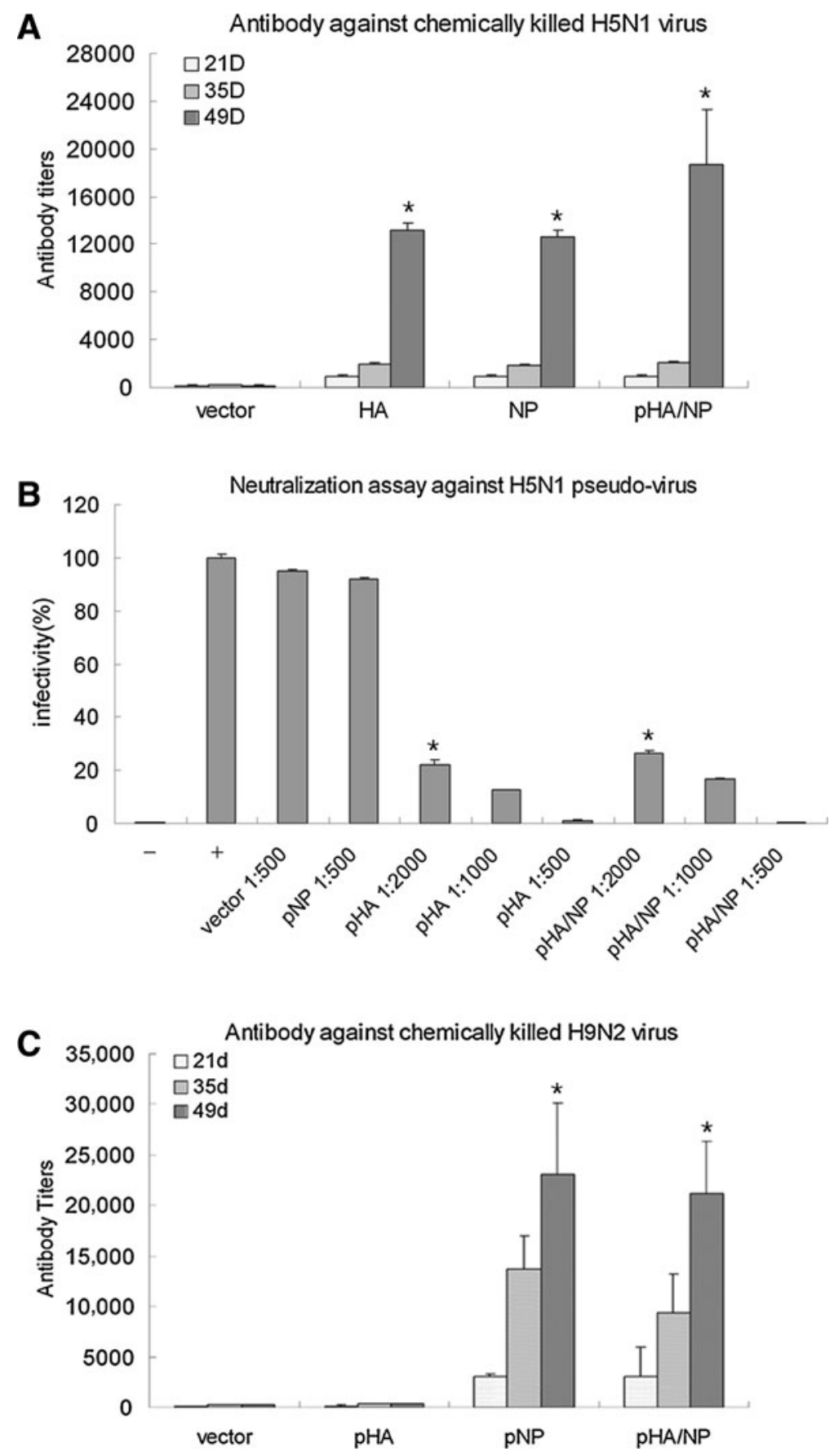

FIG. 2. Generation of anti-H5N1 homologous and anti-H9N2 heterosubtypic IgG in mice. (A) Antibody response to A/ Chicken/Henan/12/2004 (H5N1) virus. Mice were bled on days 21, 35, and 49 after the initial immunization, and sera were prepared in serial dilutions. The antibodies against the H5N1 virus were evaluated by ELISA with $5 \mu \mathrm{g} / \mathrm{mL}$ of chemicallykilled A/Chicken/Henan/12/2004 (H5N1) virus as antigen coated on each well in a 96-well plate. The titers of the sera were defined as the last serial dilution with an $\mathrm{OD}_{450}$ value exceeding the cut-off value (cut-off $=2$ times the OD value of naïve serum), and were represented as the mean titers + standard deviation in five animals $\left({ }^{*} p<0.05\right.$ by Student's $t$-test compared to the vector-immunized group). (B) Neutralizing activity of sera from immunized mice. Immunized sera were collected on day 49 after initial immunization, and prepared in serial dilutions. Neutralizing activities of the sera were determined by measuring the inhibition of H5N1 pseudotyped virus infectivity on MDCK cells (-, negative control in which no pseudotyped virus was added to the cells; + , positive control in which pseudotyped virus mixed with irrelevant antibody was added to the cells, defined as $100 \%$ infectivity; ${ }^{*} p<0.05$ compared to the vector- and pNP-immunized groups). (C) Antibody response to A/Chicken/Jiangsu/7/2002 (H9N2) virus. The antibody against the H9N2 virus was evaluated by ELISA with $5 \mu \mathrm{g} / \mathrm{mL}$ of chemically-killed A/Chicken/Jiangsu/7/2002 (H9N2) virus antigen coated on each well in a 96-well plate. The titers were calculated as above $\left({ }^{*} p<0.05\right.$ compared to the vector- and pHA-immunized groups). 
mans. Antibody titers in sera from immunized mice were determined using ELISA against chemically-killed A/Chicken/ Jiangsu/7/2002 (H9N2) virus. As shown in Fig. 2C, the bivalent $\mathrm{pHA} / \mathrm{NP}$ and the monovalent $\mathrm{pNP}$ immunizations elicited similar amounts of high-level anti-H9N2-specific antibodies, while antibody titers in both the vector- and pHAimmunized groups were quite low.

Taken together, these data suggest that only the bivalent DNA vaccine $\mathrm{pHA} / \mathrm{NP}$ was able to generate both anti-H5N1 HA-specific neutralizing antibody and anti-H9N2 NPheterosubtypic antibody.

\section{NP expression induces stronger splenocyte proliferation with a heterosubtypic response}

To determine the splenocyte response, a single suspension of splenocytes was prepared from mouse spleens on the seventh day after the third injection and assayed. As shown in Fig. 3, when stimulated with chemically-killed A/Chicken/Henan/12/2004 (H5N1) virus, cells isolated from the spleens of mice injected with both $\mathrm{pNP}$ and $\mathrm{pHA} / \mathrm{NP}$ achieved high stimulation indexes. The splenocyte proliferation in mice injected with pHA was also observable, but was relatively lower compared to the DNA constructs expressing NP protein ( $\mathrm{pNP}$ and $\mathrm{pHA} / \mathrm{NP})$, while the highest proliferation was observed in mice injected with pHA/NP. When stimulated with chemically-killed heterosubtypic A/ Chicken/Jiangsu/7/2002 (H9N2) virus, significant splenocyte proliferation was observed in mice injected with DNA constructs $\mathrm{pNP}$ or $\mathrm{pHA} / \mathrm{NP}$, but not in the $\mathrm{pHA}$ group. These data in all indicate that immunization with DNA constructs expressing NP protein ( $\mathrm{pNP}$ and $\mathrm{pHA} / \mathrm{NP}$ ) elicit more efficient and robust splenocyte proliferation responses against both homologous H5N1 and heterosubtypic H9N2 viruses.

\section{The strongest CTL response was elicited by the bivalent $\mathrm{pHA} / \mathrm{NP}$ immunization}

Virus-specific cytotoxic T lymphocyte (CTL) activity has been reported to be important in controlling virus infections via direct cytolysis of virus-infected cells. We therefore set out to determine the CTL activity using an in vivo CTL assay, which reflects more of the true stimulation than the in vitro assay. To detect the presence of anti-influenza virus-specific CTL in vivo, we adopted a previously documented method $(13,33)$. A mixture $(1: 1)$ of influenza virus major histocompatibility complex class I (MHC-I) peptides (IYSTVASSL from the HA protein and LYEKVKSQL from the NP protein), which are mostly conserved among influenza strains and subtypes, was applied to stimulate targeted splenocytes labeled with a high concentration of CFSE (CFSE $\left.{ }^{\text {high }}\right)$. The same number of untargeted splenocytes labeled with a low concentration of CFSE (CFSE ${ }^{\text {low }}$ ) was injected together with the CFSE ${ }^{\text {high }}$ cells into immunized mice intravenously. The CTL response was then evaluated, as peptide-labeled cells were killed, while antigen-unlabeled cells survived. The results in Fig. 4A show that all of the pHA, pNP, and

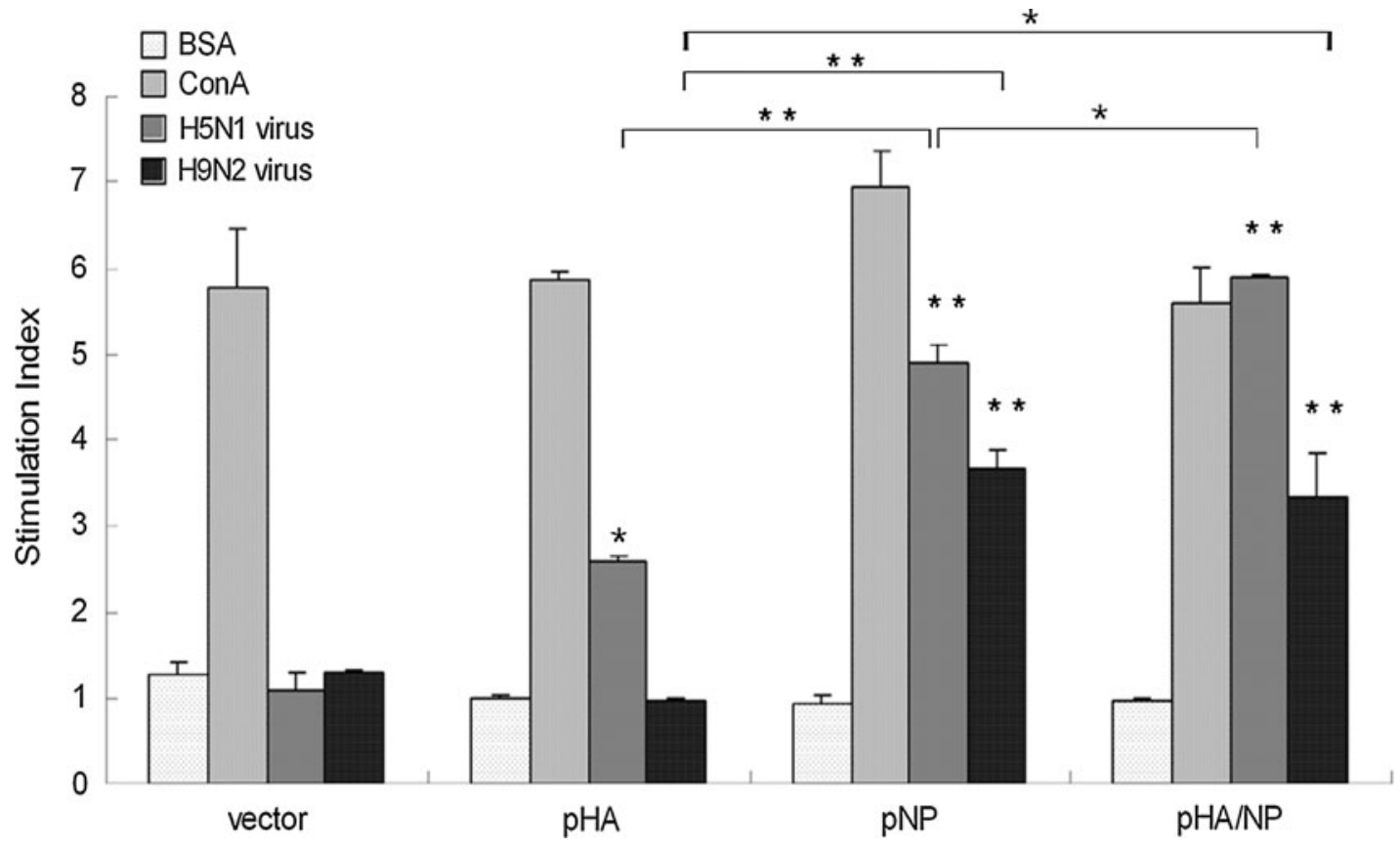

FIG. 3. T-cell proliferation after DNA vaccination. A single suspension of splenocytes of mice immunized with the indicated DNA constructs was isolated on the seventh day after the third immunization, and stimulated in vitro either with chemicallykilled A/Chicken/Henan/12/2004 (H5N1) virus, with A/Chicken/Jiangsu/7/2002 (H9N2) virus, with an irrelevant protein (BSA) as a negative control, or with ConA as a positive control. The stimulation indexes (+standard deviation) were derived as the value of the test sample divided by the value of blank medium $\left({ }^{*} p<0.05,{ }^{* *} p<0.01\right.$ compared to the BSA-stimulated index within each group; $* p<0.05, * * p<0.01$ indicate statistical significance when the indicated columns were compared between groups). 

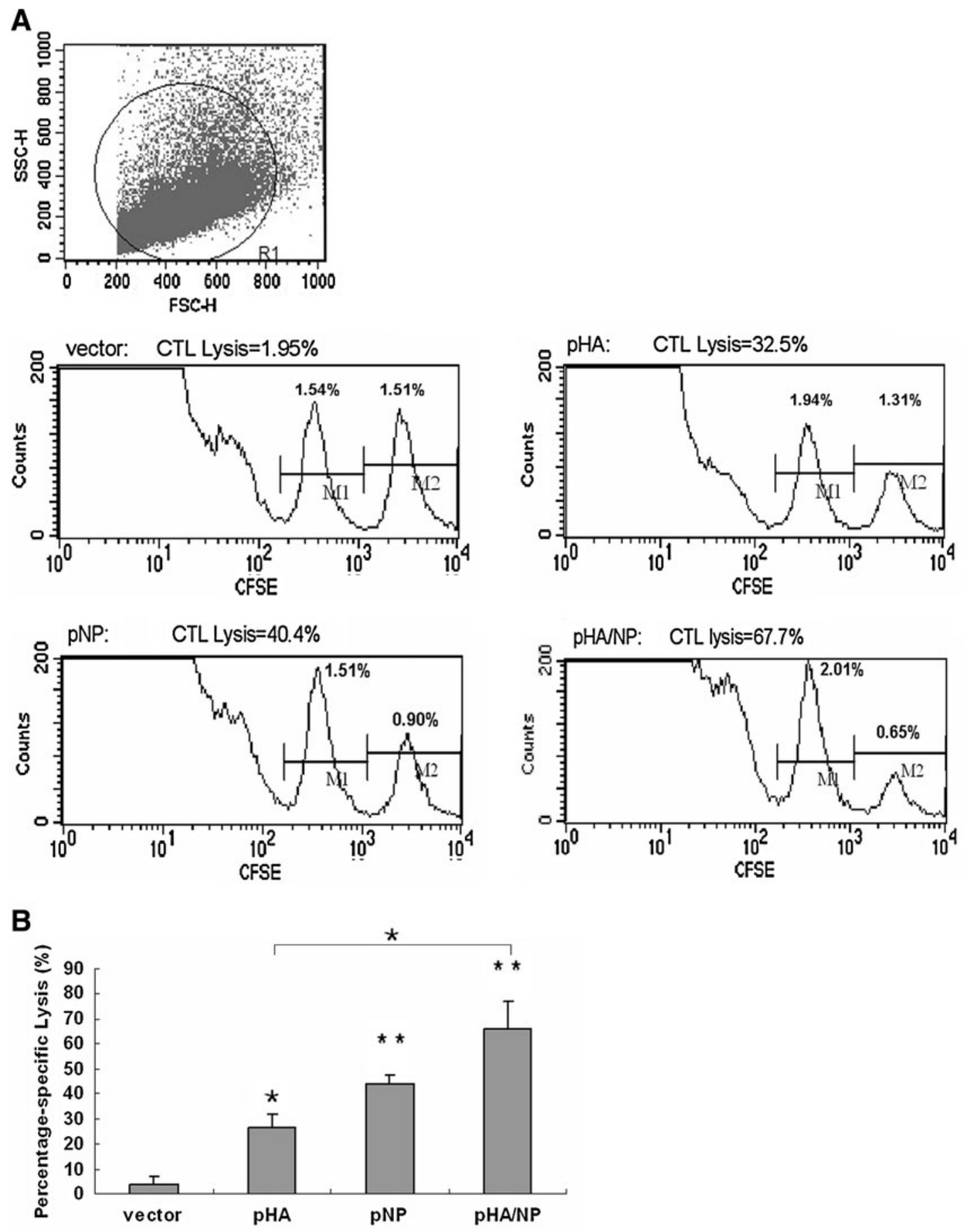

FIG. 4. In vivo influenza-virus-specific CTL assay. A 1:1 mixture of $10^{7}$ cells of each target cell population was injected intravenously into mice $7 \mathrm{~d}$ after the third injection with the indicated DNA vaccines. After $4 \mathrm{~h}$, the mice were killed and the splenocytes were analyzed for the presence of CFSE ${ }^{\text {high }}$ (pulsed with influenza epitopes), and CFSE ${ }^{\text {low }}$ (unpulsed) target cell populations. (A) Representative histogram plot of splenocytes obtained from a mouse vaccinated with each DNA construct at $4 \mathrm{~h}$ post-transfer of CFSE-labeled target cells. (B) The percentage of specific lysis (+standard deviation) in five animals was calculated as described in the materials and methods section $\left({ }^{*} p<0.05,{ }^{* *} p<0.01\right.$ compared to the vector-immunized group; $\Gamma^{*} p<0.05$ for the $\mathrm{pHA} / \mathrm{NP}$-immunized group compared to the pHA-immunized group).

pHA/NP immunizations induced significantly efficient lysis of influenza peptide-labeled cells, compared to that seen in the vector-immunized group. The quantitative analysis in Fig. 4B further suggests that pNP immunization induced higher CTL activity than pHA immunization, while the highest CTL activity was observed in mice vaccinated with the bivalent pHA/NP construct. These data in all indicate that the strongest CTL response was elicited by the bivalent DNA vaccine $\mathrm{pHA} / \mathrm{NP}$, compared to the monovalent constructs. 
Both IL-4 and IFN- $\gamma$ are induced

by the bivalent $p H A / N P$ immunization

The ability of this bivalent DNA vaccine ( $\mathrm{pHA} / \mathrm{NP}$ ) to elicit both humoral and cellular immune responses was examined by analyzing its effect on the Th-1- and Th-2-stimulated cytokine response. ELISA was used to measure the levels of IFN$\gamma$, representing the Th- 1 cytokine, and IL- 4 , representing the Th- 2 cytokine, in restimulated splenocytes taken from mice on the seventh day after the third immunization. Chemicallykilled A/Chicken/Henan/12/2004 (H5N1) and A/Chicken/ Jiangsu/7/2002 (H9N2) viruses were applied as homologous and heterosubtypic antigens, respectively; mitogen concanavalin A (ConA) was used as a positive control; and BSA was used as an irrelevant control. As shown in Fig. 5, when re-stimulated with homologous $\mathrm{H} 5 \mathrm{~N} 1$ virus, the $\mathrm{pHA}, \mathrm{pNP}$, and $\mathrm{pHA} / \mathrm{NP}$ immunizations induced high levels of IFN- $\gamma$ and IL-4 secretion, compared to vector immunization. However, only the DNA constructs expressing NP protein were able to elicit a high level of IFN- $\gamma$ and IL-4 against heterosubtypic H9N2 virus. In all, the cytokine profile seen after DNA immunizations indicates that the bivalent DNA vaccine $\mathrm{pHA} / \mathrm{NP}$ elicits high levels of both IFN- $\gamma$ and IL-4 against both homologous and heterosubtypic virus.
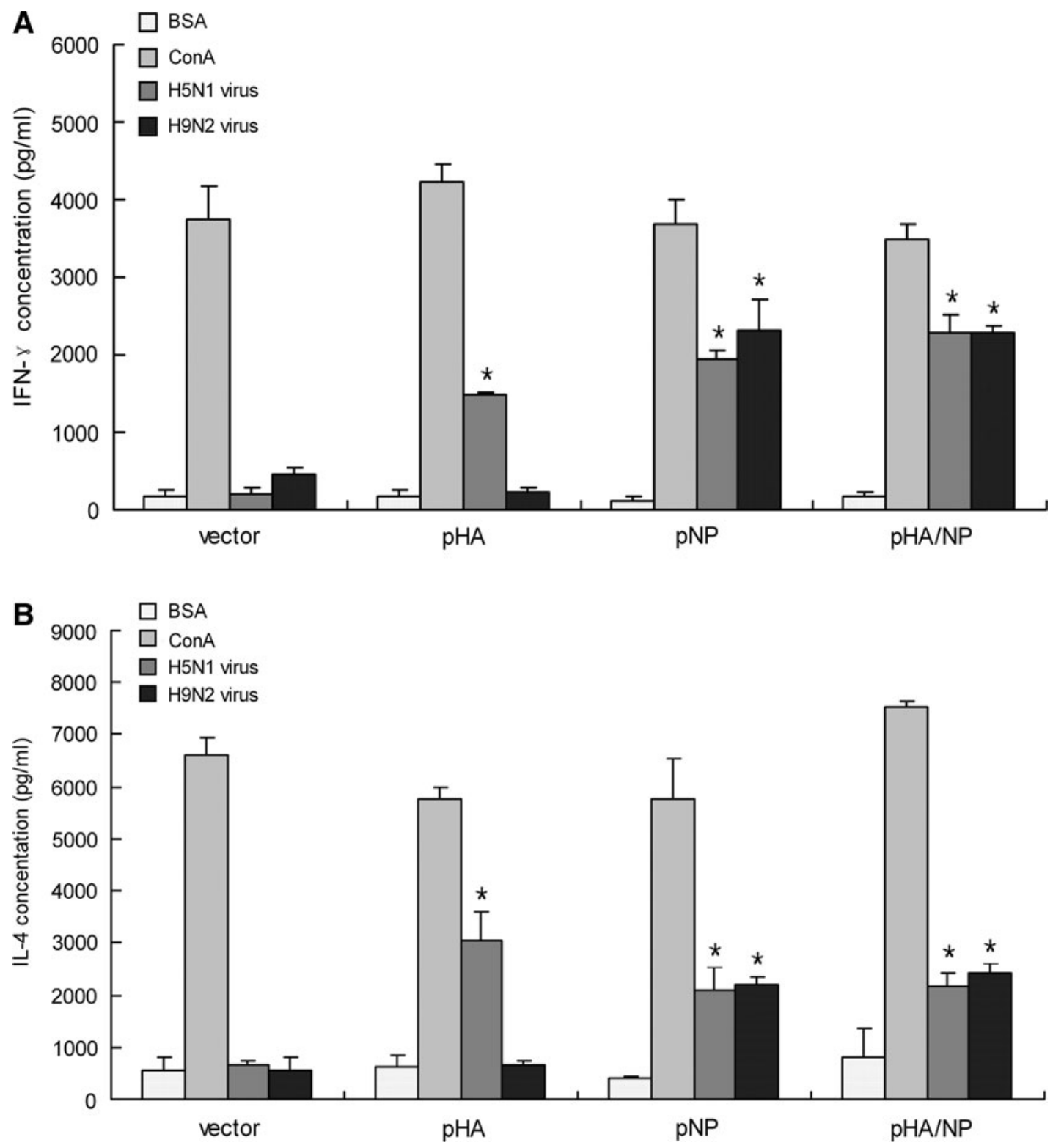

FIG. 5. Effect of DNA vaccinations on IFN- $\gamma$ and IL-4 release. Splenocytes harvested on the seventh day after the third immunization were stimulated in vitro, with chemically-killed A/Chicken/Henan/12/2004 (H5N1) virus, A/Chicken/ Jiangsu/7/2002 (H9N2) virus, with an irrelevant protein (BSA) as a negative control, or with ConA as a positive control. Cytokine ELISA detection kits were used to evaluate IFN- $\gamma$ (A) and IL-4 (B) levels. The concentrations of the released cytokines (+standard deviation) were calculated using a cytokine standard curve on the same measuring plate ${ }^{*} p<0.05$ by Student's $t$-test compared to the BSA-stimulated value). 


\section{Discussion}

Of the vaccine types against influenza virus, DNA vaccines are among the most promising vaccines, as they are capable of inducing both humoral and cellular immune responses. In this study, we developed a bivalent DNA vaccine encoding both the HA and NP proteins from a HPAI H5N1 virus. The combination of strain-specific surface viral protein, and conserved internal viral protein, makes the bivalent DNA vaccine capable of responding against both homologous and heterosubtypic viruses. This may be a potential vaccine candidate for use against both HPAI H5N1 viruses and any unexpected newly-emerging strains.

To design a DNA vaccine against influenza A virus, the surface viral protein $\mathrm{HA}$ is widely considered to be the predominant immunogen for the induction of strain-specific antibodies to prevent infection. However, with its many variations, the HA region alone can provide only weak broadly-protective immune activity (26), while the internal viral proteins, which are less variable, are reported to provide cross-subtype CTL-mediated protection $(24,46)$. Therefore, a combination of surface and internal proteins should be both effective against the current circulating strain, and protective against other antigenic variants. The first evidence for this idea was seen with successful protection from antigenically-distinct influenza isolates in ferrets immunized with a combination of independent DNA plasmids encoding HA and two internal conserved proteins $(19,20)$. However, few studies have followed this combination approach, possibly due to concerns about antigenic competition among viral proteins. Although a previous study showed no antigenic competition among the dissociated NA, M, and NP proteins of influenza A virus (28), no such data existed about the HA protein and internal viral proteins, when given either with independent plasmids or with one plasmid expressing multiple viral proteins. Our study with the bivalent DNA vaccine $\mathrm{pHA} / \mathrm{NP}$, expressing two viral proteins in a dualpromoter plasmid, demonstrates that there is no obvious antigenic competition between HA and NP. In most of the immune responses we have examined, bivalent DNA vaccines have exhibited relatively higher activity than any of the monovalent vaccines against the homologous $\mathrm{H} 5 \mathrm{~N} 1$ virus. Moreover, the similar immune responses seen with the bivalent $\mathrm{pHA} / \mathrm{NP}$ and the univalent $\mathrm{pNP}$ vaccines against heterosubtypic $\mathrm{H} 9 \mathrm{~N} 2$ virus also indicates that antigenic competition between HA and NP proteins is minimal. As a consequence, a combination of surface HA and internal NP genes in a DNA vaccine is able to elicit both strain-specific and cross-subtype immune responses without inducing antigenic competition.

Among the delivery methods of DNA vaccination, the highest efficacy was achieved by in vivo electroporation and gene gun delivery (49). Electroporation of DNA vaccines in vivo has been proven to be an effective method to increase antigen expression and to improve immune responses in many animal models $(2,38,42,53)$. In vivo electroporation has also been reported to successfully deliver influenza DNA vaccine against $\mathrm{HPAI} \mathrm{H} 5 \mathrm{~N} 1$ virus in mice $(9,58)$. In this study, in vivo electroporation was used to deliver the bivalent DNA vaccine with optimized parameters (8). Our results showed that high levels of both humoral and cellular immune responses were induced by the electroporation of bivalent
DNA vaccine without any adjuvant. Therefore, results from both previous and our studies provide promising evidence that electroporation appears to overcome the cellular uptake barrier of DNA vaccines.

To evaluate the neutralizing antibody response induced by our bivalent DNA vaccine, a pseudotyped virus-based assay was developed to avoid the need for the use of highly pathogenic H5N1 live virus. The conventional methods used to estimate neutralizing antibody responses against influenza viruses include the microneutralization (MN) and hemagglutination inhibition (HI) assays. However, both the $\mathrm{MN}$ and $\mathrm{HI}$ assays have their shortcomings. The MN assay requires the use of highly-pathogenic infectious virus, and therefore requires biosafety level 3 facilities, and is thus labor-intensive. Moreover, when evaluating the cytopathic effects, the accuracy of the MN assay is prone to the subjectivity of the researcher. With the HI assay, although it is easier to perform, differences in erythrocyte origins will cause differing $\mathrm{HI}$ titers. The lentiviral pseudotyped virus has been applied to evaluate the neutralizing antibody response against HPAI viruses $(32,43,56)$. As the pseudotyped virus undergoes a single round of infection and cannot produce progeny viruses, highly-biosafe facilities are not required. Further, the pseudotyped virus contains the luciferase reporter gene, and infectivity can thus easily be measured by reading luminescence values. Moreover, the neutralizing antibody response evaluated using pseudotyped virus-based assays have also been found to be comparable to or even more sensitive than the MN and HI assays (43). In this study, the pseudotyped virus was used to express the HA and NA proteins from the same H5N1 strain to design a DNA vaccine; we found that both $\mathrm{pHA}$ and $\mathrm{pHA} /$ NP vaccinations elicited high levels of neutralizing antibody. This result indicates that DNA vaccines expressing the influenza HA protein are able to induce neutralizing antibodies with high efficacy.

To evaluate the heterosubtypic immune response induced by the bivalent DNA vaccine, we examined both the antiheterosubtypic humoral antibody response, and the cellmediated splenocyte proliferation and CTL response. For detection of anti-heterosubtypic antibody, chemically-killed A/Chicken/Jiangsu/7/2002 (H9N2) virus was used as antigen, and a high level of anti-NP antibody was observed, indicating an efficient cross-subtype humoral immune response. A previous study indicated that recombinant NP vaccination was able to generate protective anti-NP antibodies that reduced influenza virus titers and reduced mortality in mice (5). Although NP antibody may not protect cells against influenza infection (39), the substantial antibody response generated against NP protein by natural infection or vaccination indicates a potential role for anti-NP antibody in disease control and virus clearance (14). Considering that $\mathrm{NP}$ is also expressed on the surface of influenza-infected cells, and is a major target for CTL $(35,39,48)$, it is reasonable to propose a significant role for the NP antibody in a crosssubtype immune response, perhaps even in humans. For the in vivo CTL assay, conserved HA and NP epitope-pulsed splenocytes were used as target cells to represent a general CTL response against influenza A virus. Compared to pHA, vaccination with either $\mathrm{pNP}$ and $\mathrm{pHA} / \mathrm{NP}$ induced stronger cell-mediated immune responses, including splenocyte proliferation and a CTL response against both types of viruses. 
These results are consistent with those of previous studies, indicating that influenza's internal proteins, particularly NP protein, are major targets for cell-mediated immunity (57). Both the anti-NP antibody and NP-induced cell-mediated immune responses may provide cross-reactive anti-influenza A virus protection.

The production of vaccines against HPAI viruses poses safety problems with regard to vaccine manufacture (55). The HPAI virus, like the avian H5N1 virus, kills chicken embryos in short order, resulting in substantial reductions in vaccine yield and potential contamination by the dead eggs. More seriously, production staff would be immunologically naïve to an avian virus, and thus would be susceptible to infection. Although the reverse-genetics technique based on plasmid rescue of infectious virions could create nonpathogenic vaccine strains by excising the extra basic amino acids at the cleavage site of the avian HA segment (41), relatively low efficiency in recombinant virus growth and the large demand for chicken embryos remain problematic in maintaining the global vaccine supply. Plasmid DNA vaccines are capable of overcoming all of the above problems, allowing for easier manipulation and faster production compared to traditional influenza vaccines. Several DNA vaccines against $\mathrm{H} 5 \mathrm{~N} 1$ virus have been described, using either HA-expressing plasmid, or internal M- and NP-expressing plasmids $(9,22,29)$, though few studies have combined the surface and internal viral proteins to study the heterosubtypic immune response. In this study, we constructed a bivalent DNA vaccine simultaneously expressing HA and $\mathrm{NP}$ proteins from an HPAI H5N1 virus. The surface HA protein expressed from the bivalent DNA vaccine induces an anti-H5N1-specific neutralizing antibody that can prevent viral infection, while the internal conserved NP protein expressed from the bivalent DNA vaccine induces both homologous and heterosubtypic anti-NP antibody, and cellmediated immune responses that can aid in virus clearance. One can thus predict that the combined expression of influenza HA and NP by a bivalent DNA vaccine may be a new strategy to achieve protection against homo-subtypic influenza virus infection, as well as cross-protection against hetero-subtypic infections. This bivalent DNA vaccine may be a new vaccine candidate for use in the event of an HPAI pandemic.

\section{Acknowledgments}

This work was supported by a Chinese Academy of Sciences project (KSCX2-YW-R-161), a grant from the National Ministry of Science and Technology (20072714), grants from the National Natural Science Foundation of China (30950002, 30623003, 30721065, 30801011, 30870126, and 90713044), grants from the Science and Technology Commission of Shanghai Municipality (08DZ2291703, 088014199, and 08431903004), a National Science and Technology Major Project (2008ZX10002-014, 2008ZX10004-002, and 2009ZX10004-105), a grant from the National 973 key project (2007CB512404), grants from Shanghai Pasteur Health Research Foundation (SPHRF2008001 and SPHRF2009001), the National 863 project (2006AA02A247), and a grant from the Li Kha Shing Foundation.

\section{Author Disclosure Statement}

No competing financial interests exist.

\section{References}

1. Aihara $\mathrm{H}$, and Miyazaki J: Gene transfer into muscle by electroporation in vivo. Nat Biotechnol 1998;16:867-870.

2. Babiuk S, Baca-Estrada ME, Foldvari M, Storms M, Rabussay D, Widera G, and Babiuk LA: Electroporation improves the efficacy of DNA vaccines in large animals. Vaccine 2002;20:3399-3408.

3. Bian C, Zhang X, Cai X, et al.: Conserved amino acids W423 and N424 in receptor-binding domain of SARS-CoV are potential targets for therapeutic monoclonal antibody. Virology 2009;383:39-46.

4. Butt KM, Smith GJ, Chen H, et al.: Human infection with an avian H9N2 influenza A virus in Hong Kong in 2003. J Clin Microbiol 2005;43:5760-5767.

5. Carragher DM, Kaminski DA, Moquin A, Hartson L, and Randall TD: A novel role for non-neutralizing antibodies against nucleoprotein in facilitating resistance to influenza virus. J Immunol 2008;181:4168-4176.

6. Cauthen A, Swayne D, Schultz-Cherry S, Perdue M, and Suarez D: Continued circulation in China of highly pathogenic avian influenza viruses encoding the hemagglutinin gene associated with the $1997 \mathrm{H} 5 \mathrm{~N} 1$ outbreak in poultry and humans. J Virol 2000;74:6592-6599.

7. Centers for Disease Control and Prevention: Swine influenza (H1N1) infection in two children. MMWR Morb Mortal Wkly Rep 2009;58:400-402.

8. Chen J, Fang F, Li X, Chang H, and Chen Z: Protection against influenza virus infection in BALB/c mice immunized with a single dose of neuraminidase-expressing DNAs by electroporation. Vaccine 2005;23:4322-4328.

9. Chen MW, Cheng TJ, Huang Y, et al.: A consensus-hemagglutinin-based DNA vaccine that protects mice against divergent H5N1 influenza viruses. Proc Natl Acad Sci USA 2008;105:13538-13543.

10. Chen Z, Kadowaki S, Hagiwara Y, Yoshikawa T, Matsuo K, Kurata T, and Tamura S: Cross-protection against a lethal influenza virus infection by DNA vaccine to neuraminidase. Vaccine 2000;18:3214-3222.

11. Chen Z, Zhou P, Ho DD, Landau NR, and Marx PA: Genetically divergent strains of simian immunodeficiency virus use CCR5 as a coreceptor for entry. J Virol 1997;71:27052714.

12. Claas ECJ: Human influenza A H5N1 virus related to a highly pathogenic avian influenza virus. Lancet 1998;351: 472-477.

13. Coles RM, Mueller SN, Heath WR, Carbone FR, and Brooks AG: Progression of armed CTL from draining lymph node to spleen shortly after localized infection with herpes simplex virus 1. J Immunol 2002;168:834-838.

14. Couch RB: An overview of serum antibody responses to influenza virus antigens. Dev Biol (Basel) 2003;115: 25-30.

15. Davis HL: Intramuscular and intradermal injection of DNA vaccine in mice and primates methods in molecular medicine. DNA Vaccine Methods Protocols 29.

16. De Jong JC, Claas EC, Osterhaus AD, Webster RG, and Lim WL: A pandemic warning? Nature 1997;389:554.

17. Deng Y, Yewdell JW, Eisenlohr LC, and Bennink JR: MHC affinity, peptide liberation, $\mathrm{T}$ cell repertoire, and immunodominance all contribute to the paucity of MHC class I-restricted peptides recognized by antiviral CTL. J Immunol 1997;158:1507-1515.

18. DiMenna LJ, and Ertl HC: Pandemic influenza vaccines. Curr Top Microbiol Immunol 2009;333:291-321. 
19. Donnelly JJ, Friedman A, Martinez D, et al.: Preclinical efficacy of a prototype DNA vaccine: enhanced protection against antigenic drift in influenza virus. Nat Med 1995;1:583-587.

20. Donnelly JJ, Friedman A, Ulmer JB, and Liu MA: Further protection against antigenic drift of influenza virus in a ferret model by DNA vaccination. Vaccine 1997;15:865-868.

21. Donnelly JJ, Ulmer JB, Shiver JW, and Liu MA: DNA vaccines. Annu Rev Immunol 1997;15:617-648.

22. Epstein SL, Tumpey TM, Misplon JA, et al.: DNA vaccine expressing conserved influenza virus proteins protective against H5N1 challenge infection in mice. Emerg Infect Dis 2002;8:796-801.

23. Fraser C, Donnelly CA, Cauchemez $S$, et al.: Pandemic potential of a strain of influenza A (H1N1): early findings. Science 2009;324:1557-1561.

24. Fu TM, Ulmer JB, Caulfield MJ, et al.: Priming of cytotoxic T lymphocytes by DNA vaccines: requirement for professional antigen presenting cells and evidence for antigen transfer from myocytes. Mol Med 1997;3:362-371.

25. Garten RJ, Davis CT, Russell CA, et al.: Antigenic and genetic characteristics of swine-origin 2009 A(H1N1) influenza viruses circulating in humans. Science 2009;325:197-201.

26. Gerhard W, Mozdzanowska K, and Zharikova D: Prospects for universal influenza virus vaccine. Emerg Infect Dis 2006;12:569-574.

27. Jin $\mathrm{H}, \mathrm{Xiao} \mathrm{C}, \mathrm{Chen} \mathrm{Z}$, et al.: Induction of Th1 type response by DNA vaccinations with $\mathrm{N}, \mathrm{M}$, and $\mathrm{E}$ genes against SARS$\mathrm{CoV}$ in mice. Biochem Biophys Res Commun 2005;328:979986.

28. Johansson BE, and Kilbourne ED: Immunization with dissociated neuraminidase, matrix, and nucleoproteins from influenza A virus eliminates cognate help and antigenic competition. Virology 1996;225:136-144.

29. Kodihalli S, Goto H, Kobasa DL, Krauss S, Kawaoka Y, and Webster RG: DNA vaccine encoding hemagglutinin provides protective immunity against H5N1 influenza virus infection in mice. J Virol 1999;73:2094-2098.

30. Kodihalli S, Haynes JR, Robinson HL, and Webster RG: Cross-protection among lethal H5N2 influenza viruses induced by DNA vaccine to the hemagglutinin. J Virol 1997;71:3391-3396.

31. Lin $\mathrm{YP}$, Shaw M, Gregory V, et al.: Avian-to-human transmission of H9N2 subtype influenza A viruses: relationship between H9N2 and H5N1 human isolates. Proc Natl Acad Sci USA 2000;97:9654-9658.

32. Nefkens I, Garcia JM, Ling CS, et al.: Hemagglutinin pseudotyped lentiviral particles: characterization of a new method for avian H5N1 influenza sero-diagnosis. J Clin Virol 2007;39:27-33.

33. Oehen S, and Brduscha-Riem K: Differentiation of naive CTL to effector and memory CTL: correlation of effector function with phenotype and cell division. J Immunol 1998;161:53385346.

34. Palese P, and Garcia-Sastre A: Influenza vaccines: present and future. J Clin Invest 2002;110:9-13.

35. Prokudina EN, and Semenova NP: Localization of the influenza virus nucleoprotein: cell-associated and extracellular non-virion forms. J Gen Virol 1991;72(Pt 7):1699-1702.

36. Riedel S: Crossing the species barrier: the threat of an avian influenza pandemic. Proc (Bayl Univ Med Cent) 2006;19:1620.

37. Robinson HL, Hunt LA, and Webster RG: Protection against a lethal influenza virus challenge by immunization with a haemagglutinin-expressing plasmid DNA. Vaccine 1993;11: 957-960.

38. Scheerlinck JP, Karlis J, Tjelle TE, Presidente PJ, Mathiesen I, and Newton SE: In vivo electroporation improves immune responses to DNA vaccination in sheep. Vaccine 2004;22: 1820-1825.

39. Stitz L, Schmitz C, Binder D, Zinkernagel R, Paoletti E, and Becht $\mathrm{H}$ : Characterization and immunological properties of influenza A virus nucleoprotein (NP): cell-associated NP isolated from infected cells or viral NP expressed by vaccinia recombinant virus do not confer protection. J Gen Virol 1990;71(Pt 5):1169-1179.

40. Subbarao, K: Characterization of an avian influenza A (H5N1) virus isolated from a child with a fatal respiratory illness. Science 1998;279:393-396.

41. Subbarao K, Chen H, Swayne D, et al.: Evaluation of a genetically modified reassortant $\mathrm{H} 5 \mathrm{~N} 1$ influenza A virus vaccine candidate generated by plasmid-based reverse genetics. Virology 2003;305:192-200.

42. Tollefsen S, Tjelle T, Schneider J, et al.: Improved cellular and humoral immune responses against Mycobacterium tuberculosis antigens after intramuscular DNA immunisation combined with muscle electroporation. Vaccine 2002;20: 3370-3378.

43. Tsai C, Caillet $\mathrm{C}, \mathrm{Hu} \mathrm{H}$, et al.: Measurement of neutralizing antibody responses against $\mathrm{H} 5 \mathrm{~N} 1$ clades in immunized mice and ferrets using pseudotypes expressing influenza hemagglutinin and neuraminidase. Vaccine 2009;27:67776790.

44. Ulmer JB: Influenza DNA vaccines. Vaccine 2002;20(Suppl 2):S74-S76.

45. Ulmer JB, Deck RR, DeWitt CM, Friedman A, Donnelly JJ, and Liu MA: Protective immunity by intramuscular injection of low doses of influenza virus DNA vaccines. Vaccine 1994;12:1541-1544.

46. Ulmer JB, Donnelly JJ, Parker SE, et al.: Heterologous protection against influenza by injection of DNA encoding a viral protein. Science 1993;259:1745-1749.

47. Ulmer JB, Fu TM, Deck RR, et al.: Protective CD4+ and CD8+ $\mathrm{T}$ cells against influenza virus induced by vaccination with nucleoprotein DNA. J Virol 1998;72:56485653.

48. Virelizier JL, Allison AC, Oxford JS, and Schild GC: Early presence of ribonucleoprotein antigen on surface of influenza virus-infected cells. Nature 1977;266:52-54.

49. Wells DJ: Gene therapy progress and prospects: electroporation and other physical methods. Gene Ther 2004;11: 1363-1369.

50. World Health Organization: Cumulative number of confirmed human cases of avian influenza A/(H5N1) Reported to WHO, 2010. http://www.who.int/csr/disease/avian_ influenza/country/en/.

51. World Health Organization: New influenza (H1N1) virus infections: global surveillance summary. Wkly Epidemiol Rec 2009;84.

52. World Health Organization: Recommended composition of influenza virus vaccines for use in the 2009-2010 influenza season, 2009. http://www.who.int/csr/disease/influenza/ 200902_recommendation.pdf.

53. Widera G, Austin M, Rabussay D, et al.: Increased DNA vaccine delivery and immunogenicity by electroporation in vivo. J Immunol 2000;164:4635-4640.

54. Wong SS, and Yuen KY: Avian influenza virus infections in humans. Chest 2006;129:156-168. 
55. Wood JM, and Robertson JS: From lethal virus to life-saving vaccine: developing inactivated vaccines for pandemic influenza. Nat Rev Microbiol 2004;2:842-847.

56. Yang ZY, Wei CJ, Kong WP, Wu L, Xu L, Smith DF, and Nabel GJ: Immunization by avian H5 influenza hemagglutinin mutants with altered receptor binding specificity. Science 2007;317:825-828.

57. Yewdell JW, Bennink JR, Smith GL, and Moss B: Influenza A virus nucleoprotein is a major target antigen for cross-reactive anti-influenza A virus cytotoxic T lymphocytes. Proc Natl Acad Sci USA 1985;82:1785-1789.

58. Zheng L, Wang F, Yang Z, Chen J, Chang H, and Chen Z: A single immunization with HA DNA vaccine by electroporation induces early protection against H5N1 avian influenza virus challenge in mice. BMC Infect Dis 2009;9:17.

Address correspondence to: Dr. Bing Sun Laboratory of Molecular Virology Institute Pasteur of Shanghai Chinese Academy of Sciences 225 South Chongqing Road Shanghai, 200025, China

E-mail: bsun@sibs.ac.cn

Received May 17, 2010; accepted September 12, 2010. 